\title{
INVESTIGATIONS OF STELLAR CORONAE WITH XMM-NEWTON
}

\author{
M. Audard ${ }^{1}$ \\ ${ }^{1}$ Columbia Astrophysics Laboratory, Mail code 5247, 550 West $120^{\text {th }}$ Street, New York, NY 10027, USA
}

\begin{abstract}
The XMM-Newton observatory has given a strong new impetus to the investigations of stellar coronae. It carries high-resolution X-ray spectroscopic instruments that give access to information on the physical conditions in coronal plasmas. Furthermore, its imaging detectors produce high-quality X-ray light curves and images with a large field of view. I review results on stellar coronae obtained with XMM-Newton between its launch in late 1999 and the present (late 2002). Among other topics, I discuss the elemental composition of stellar coronae and plasma electron densities in quiescence and during flares. I present evidence for various signatures of the Neupert effect in stars, which provides evidence of chromospheric and coronal heating by flares. Coronal structures based on eclipse mapping inferred from light curve inversion are shown.
\end{abstract}

\section{INTRODUCTION}

Since the discovery of X-ray emission from the first coronal source other than the Sun, Capella (Catura et al., 1975), the interpretation of physical mechanisms in stellar coronae based on X-ray data has been a rich and prosperous field. Previous X-ray satellites have shown that coronae exist in almost every late-type star (Linsky, 1985). Xray spectroscopy has played an important role in investigating stellar coronae. Previous instruments however either lacked high spectral resolution (e.g., ROSAT, ASCA, BeppoSAX), or large effective areas (e.g., EUVE). The launches of Chandra and XMM-Newton in 1999 gave access to a new approach for the study of stellar coronae: high-resolution X-ray spectroscopy with large effective areas can now be routinely obtained from 1 to $180 \AA$, thus providing us with direct access to plasma electron densities, elemental abundances, temperature structures, and this without much spectral confusion. While Chandra's strengths lie in its excellent angular resolution $\left(\approx 0.5^{\prime \prime}\right)$ and its very high spectral resolution (up to $E / \Delta E=1000$ ), XMM-Newton's strengths lie in its larger effective area (of its grating spectrometers and its CCD cameras) and its simultaneous monitoring with a total of 6 scientific instruments in the optical/UV and Xrays. XMM-Newton provides high-resolution X-ray spectra with a large signal-to-noise ratio, with a spectral resolution $(E / \Delta E \approx 100-500)$ sufficient to resolve many emission lines.

Schmelz (2003, this issue) quotes an internal US election campaign slogan to emphasize why stellar astronomers should be interested in the Sun, and modifies it to "It's the resolution, stupid!", implicitly mentioning the spatial resolution. Since stars cannot be resolved with current instruments, spatial resolution cannot be an issue. Therefore, why do stellar astronomers admire the novelty of Chandra and XMM-Newton, with respect to previous X-ray instruments? "It's the spectral resolution, stupid!"

This review aims at giving a summary of the status of coronal studies with XMM-Newton as of late 2002. With the advent of new missions, it is often malpractice to "forget" about the numerous contributions from previous missions. In this review, I have tried to provide some references. A more complete introduction to the pre-XMM-Newton and Chandra era can be found in numerous reviews about stellar coronal emission (e.g. Rosner et al., 1985; Pallavicini et al., 1989; Mewe, 1991; Haisch and Schmitt, 1996; Mewe, 1996). Furthermore, this review presents, by definition, a biased view of coronal investigations based on XMM-Newton observations; thus the interested reader is referred to the similar review about Chandra results (Linsky, 2003, this issue). 

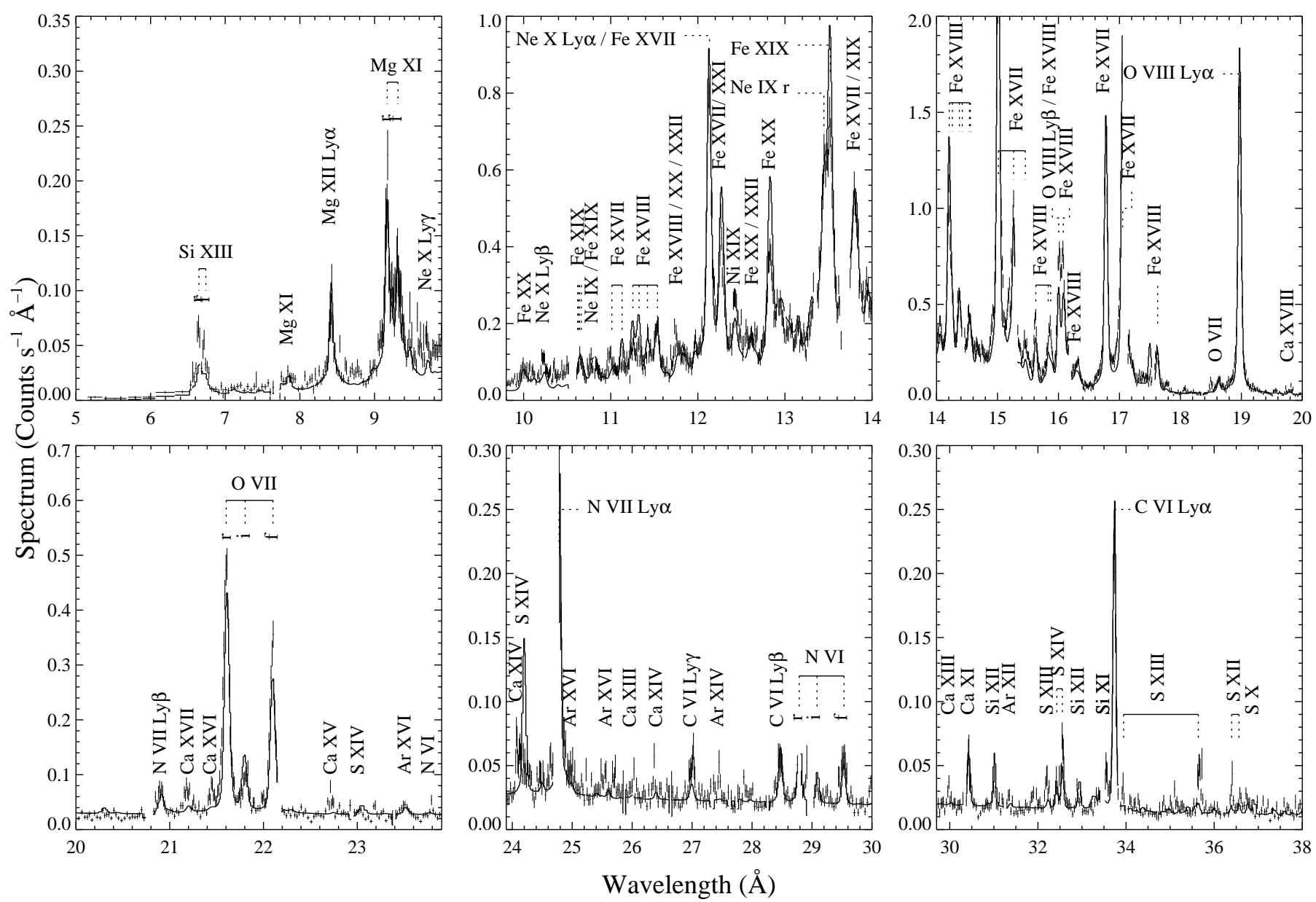

Fig. 1. XMM-Newton RGS1 spectrum of Capella together with a best-fit model. Note the diversity of elements and ionization stages and the different vertical axes. Adapted from Audard et al. (2001a), used with permission of Astron. Astrophys.

After three years of successful observations, XMM-Newton has produced excellent science; it is a difficult task to report the results of more than 15 refereed papers (published or in press) in less than 10 pages. I have thus covered three topics that broadly encompass the XMM-Newton publications: i) the elemental composition of stellar coronae; ii) the physics of stellar flares; and iii) coronal structures.

\section{XMM-NEWTON AND ITS SCIENTIFIC INSTRUMENTS}

The XMM-Newton satellite was launched with an Ariane V rocket on 10 December 1999 from the European Space Agency's launch site in Kuru, French Guyana (Jansen et al., 2001). XMM-Newton carries three X-ray telescopes consisting of 58 nested Wolter I mirrors. Behind two of the X-ray telescopes, Reflection Grating Arrays (RGA) have been placed to disperse about half of the incident X-ray light which falls on nine CCD cameras. The other half of the light is focused on MOS-type CCD arrays. The third X-ray telescope focuses the whole X-ray light onto an array of pn-type CCDs. The three CCD arrays are part of the European Photon Imaging Cameras (EPIC; Turner et al., 2001; Strüder et al., 2001). The two RGAs and 18 CCDs define the Reflection Grating Spectrometers (RGS; den Herder et al., 2001). XMM-Newton also carries an optical/UV telescope, the Optical Monitor (OM; Mason et al., 2001). All $\mathrm{X}$-ray and optical instruments can operate simultaneously with several possible modes and filters.

The RGS have high spectral resolution $(E / \Delta E=100-500)$ in the soft X-ray range $(6-38 \AA$, or $0.3-2.1 \mathrm{keV})$ with an effective area peaking at $140 \mathrm{~cm}^{2}$ around $15 \AA$. This wavelength range contains numerous $\mathrm{K}$-shell transitions of $\mathrm{C}, \mathrm{N}, \mathrm{O}, \mathrm{Ne}, \mathrm{Mg}$, and $\mathrm{Si}$, and $\mathrm{L}$-shell transitions of $\mathrm{Fe}$ and Ni. The EPIC provide access to a broad energy range $(0.15-15 \mathrm{keV})$ with large effective areas (peaking around $\left.1500 \mathrm{~cm}^{2}\right)$, however with moderate spectral resolution $(E / \Delta E=20-50)$. Their angular resolution is also moderate ( 6 " FWHM; $\left.15^{\prime \prime} \mathrm{HEW}\right)$ for a field of view (FOV) of 

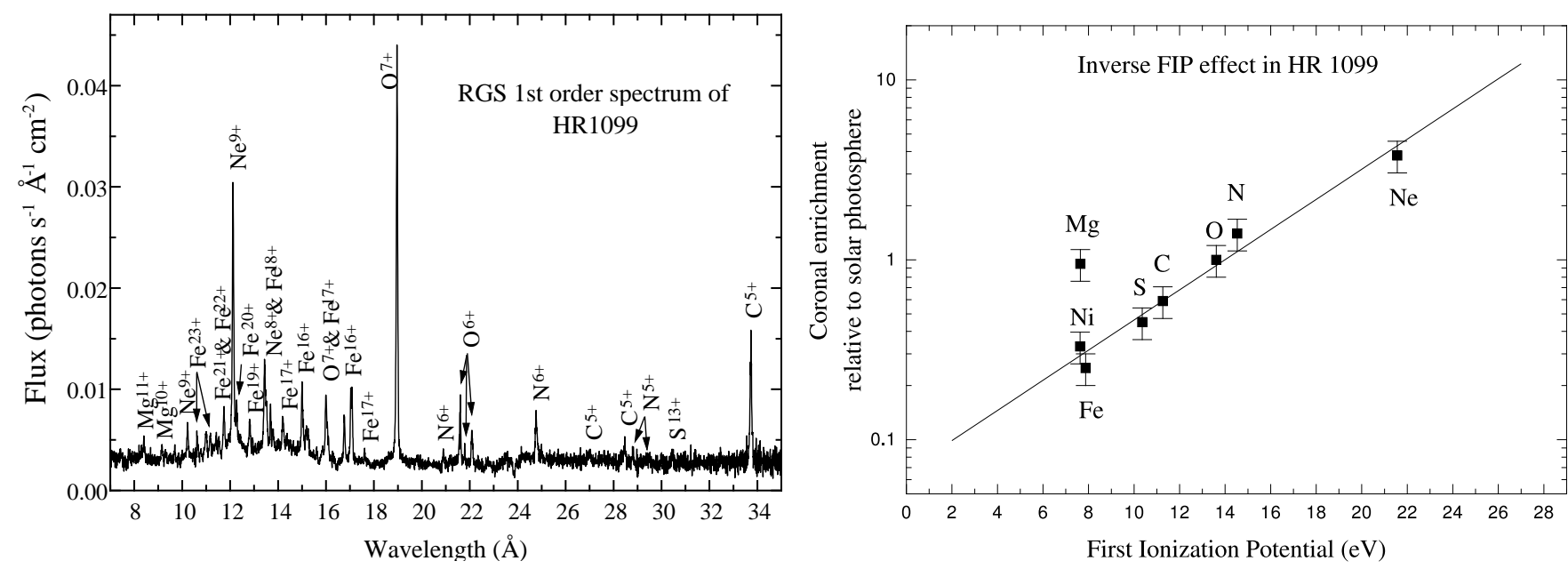

Fig. 2. Left: RGS spectrum of HR 1099. Right: Coronal abundances relative to solar photospheric abundances versus the first ionization potential. High-FIP elements are overabundant with respect to the low-FIP elements, indicating an inverse FIP effect. From Brinkman et al. (2001), used with permission of Astron. Astrophys.

$30^{\prime}$. Filters can be introduced to reduce contamination by optical photons at low energies. The EPIC can also operate with different imaging or timing modes. The OM uses a CCD intensified by a microchannel plate. It carries filters from the optical band to the ultraviolet band $\left(170 \mathrm{~nm}\right.$ to $650 \mathrm{~nm}$ ). Its FOV covers the central $17^{\prime}$ square region of the $\mathrm{X}$-ray FOV, thus permitting simultaneous multiwavelength observations.

\section{THE ELEMENTAL COMPOSITION OF STELLAR CORONAE}

The wavelength range of the RGS includes a rich spectrum of bright emission lines from several elements. Figure 1 shows the high signal-to-noise ratio spectrum of Capella which exemplifies the richness of spectral information with the RGS. Since coronal plasmas are consistent with the optically thin regime (e.g., Audard et al., 2003), the elemental composition of the coronal plasma in stars can be derived from spectral modeling. However, the current uncertainties in atomic parameters (e.g., Laming, 2002), especially of low-Z L-shell lines (e.g., Ca, Ar, Si, S), can lead to considerable bias if the spectral fit is not carried out properly. Audard et al. (2001a, 2003) showed that it is probably best, at this moment, to discard spectral ranges where bright low-Z L-shell lines are found. Future improvements in the spectral codes will eventually stop this auto-da-fé, and allow us to use the full range of data.

The study of abundances in stellar coronae has been a lively topic with previous satellites. A deficiency of metal abundance, or $\mathrm{Fe}$, by factors of 5 to 10 relative to solar photospheric abundances was observed in stars with high levels of magnetic activity (e.g., Schmitt et al., 1996). Such behavior contrasted dramatically with the solar First Ionization Potential (FIP) Effect: elements with a low FIP $(<10 \mathrm{eV})$ are overabundant by factors of $4-6$ relative to their respective photospheric abundances, while high-FIP elements are of photospheric composition (Feldman, 1992; Meyer, 1985). High-resolution spectroscopic studies with EUVE observed a solar-like FIP effect in inactive stars (Drake et al., 1997; Laming and Drake, 1999).

From a deep XMM-Newton RGS exposure of the RS CVn binary HR 1099, Brinkman et al. (2001) obtained the coronal abundances of several elements ( $\mathrm{Ne}, \mathrm{N}, \mathrm{O}, \mathrm{C}, \mathrm{S}, \mathrm{Fe}, \mathrm{Mg}$, and $\mathrm{Ni}$; Figure 2 left) and observed a peculiar abundance pattern as a function of the FIP (Figure 2 right): they found evidence for an inverse FIP effect, i.e., the abundances (relative to oxygen) increase with increasing FIP. Chandra observations of the same star confirmed the high Ne/Fe ratio (Drake et al., 2001). Other active stars showed an abundance pattern compatible with the inverse FIP effect (AB Dor: Güdel et al., 2001a; YY Gem: Güdel et al., 2001b; Stelzer et al., 2002), although the less active Capella did not show evidence for a FIP-related bias (Audard et al., 2001a).

A systematic study of coronal abundances in RS CVn binaries (HR 1099, UX Ari, $\lambda$ And, VY Ari, and Capella) confirmed that the most active binaries showed a marked inverse FIP effect, while no FIP-related bias could be found in the abundance pattern in the less active Capella (Figure 3 left, Audard et al., 2003). The abundance ratios were found to be insensitive to the spectral code chosen (XSPEC/APEC: Arnaud, 1996; Smith et al., 2001, or SPEX/MEKAL: 

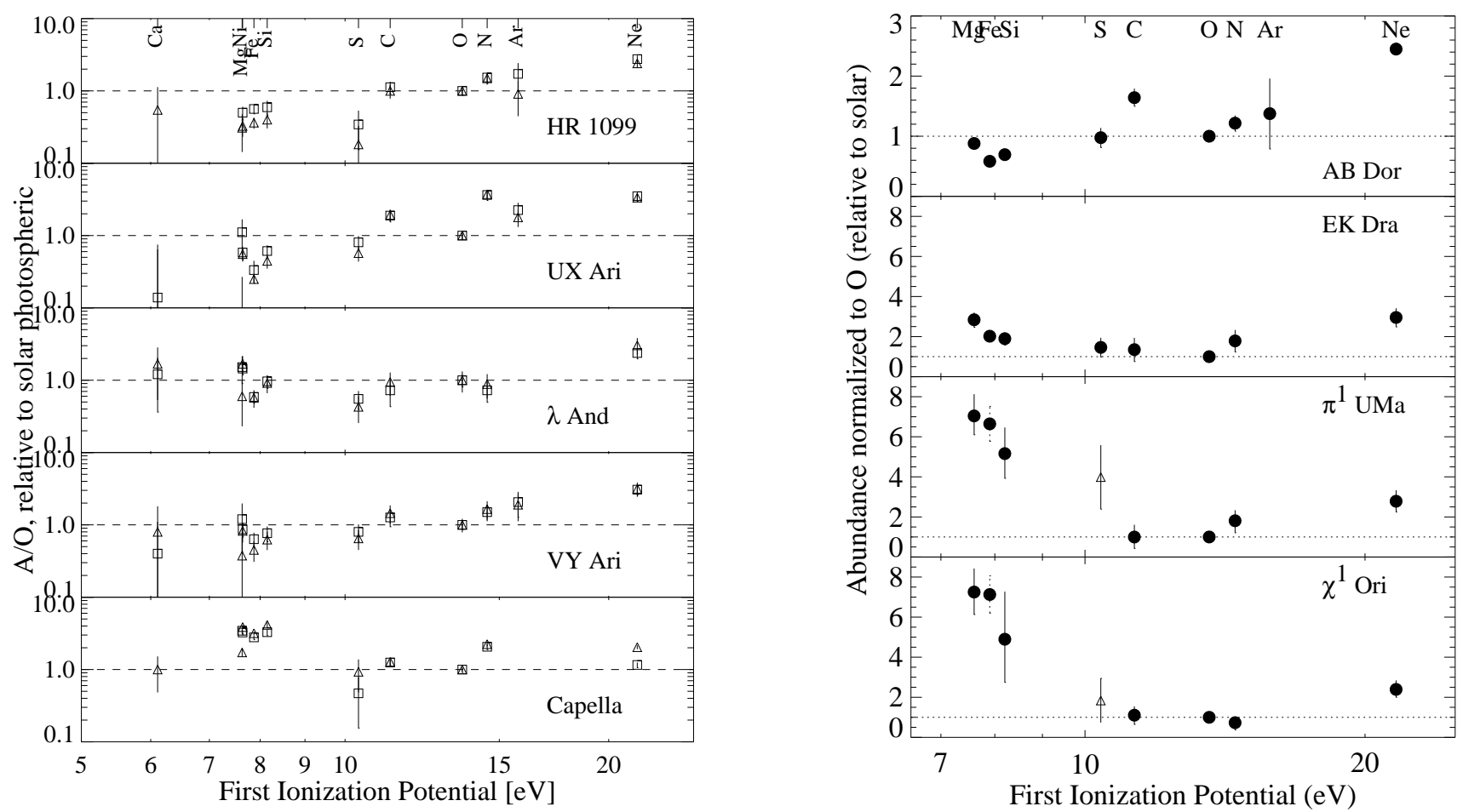

Fig. 3. Left: Coronal abundances relative to solar photospheric abundances and normalized to oxygen for five RS CVn binaries (Audard et al., 2003). The activity level decreases from top to bottom. Abundance ratios from fits with XSPEC/APEC (triangles) and SPEX/MEKAL (squares) are given. Right: Similar, but for four solar analogs (Güdel et al., 2002a). Abundance ratios from XSPEC/APEC fits only are shown. Used with permission of Astron. Astrophys. and the American Astronomical Society.

Kaastra et al., 1996; Mewe et al., 1995; Phillips et al., 1999), although "absolute" abundances (relative to solar) could potentially depend on the proper determination of the underlying continuum. Audard et al. (2003) found that the FIP-related abundance pattern did not disappear when more recent solar photospheric values were used, although its shape resembled a two-plateau with a transition around $10 \mathrm{eV}$ rather than a power law.

The observed trend may however suffer from the inappropriate set of photospheric abundances; indeed, it is common practice to normalize coronal abundances in stars with photospheric abundances in the Sun, since stellar photospheric abundances are often uncertain or even unknown. Güdel et al. (2002a) circumvented this problem and studied the coronal composition in solar analogs for which the photospheric composition is thought to be solar. They showed that a transition occurs from an inverse FIP effect in the most active (and youngest) solar analogs to a normal solar-like FIP effect in the least active (and older) stars (Figure 3 right). Audard et al. (2003) have merged the results from the solar analogs and the RS CVn binaries and showed a significant anti-correlation between the average coronal temperature of a star and its $\mathrm{Fe} / \mathrm{O}$ abundance ratio; they also showed that the $\mathrm{Ne} / \mathrm{O}$ ratio is, however, not temperature-sensitive.

This scheme may be too simplistic: previous observations with EUVE showed that the old, inactive Procyon displays no FIP bias at all (Drake et al. 1995), which is confirmed with XMM-Newton and Chandra LETG (Raassen et al., 2002), while a solar-like FIP effect could be expected. There is a clear need for a comprehensive study of the coronal composition in stars of all activity levels.

The apparent depletion of low-FIP elements in quiescent (not obviously flaring) coronae contrasts with the significant increase of abundances during flares. A time-dependent analysis of a flare in HR 1099 suggests that the abundances of low-FIP elements increase more significantly than those of high-FIP elements (Audard et al., 2001b). A near-photospheric mixture of elements is brought up into the corona. Increases in the Fe abundance (or in the average metallicity) has also been observed in flares in YY Gem (Güdel et al., 2001b; Stelzer et al., 2002), AB Dor (Güdel et al., 2001a), and possibly FK Com (Gondoin et al., 2002).

Some models have been proposed to explain the solar FIP effect (see Hénoux, 1995, 1998, for reviews of models 
and their caveats); future models will need to account for the inverse FIP effect observed in the most active stars. Drake (2003, this issue) reviews models attempting to explain coronal abundance patterns. Based on XMM-Newton observations, Güdel et al. (2002a) have proposed an explanation for the inverse FIP effect involving high-energy electrons. These particles are detected by their gyrosynchrotron emission as the propagate along the magnetic fields. At the upper chromospheric level, they could prevent ions (mostly low-FIP elements at chromospheric temperatures) from diffusing up into the corona by creating a downward-pointing electric field. In less active stars, in which the radio flux (and the high-energy particle density) is smaller, low-FIP elements would thus be able to escape into the corona, thus creating a solar-like FIP effect. During flares, when the density of high-energy particles is very large, chromospheric evaporation occurs and brings up near-photospheric material into the corona (Audard et al., 2001b). Although appealing, more detailed analysis and sophisticated models are needed.

Sciortino et al. (2001) studied the low-metallicity $([\mathrm{Fe} / \mathrm{H}]=-0.32$ to -0.18$)$ open cluster NGC 2516 . This cluster is coeval with the more metal rich Pleiades cluster $(\approx 100 \mathrm{Myr})$. They found that the X-ray luminosity function of NGC 2516 is less luminous than that of the Pleiades for photometrically selected G and K dwarfs, whereas it is indistinguishable for M dwarfs. Sciortino et al. (2001) argued that metallicity could explain this behavior by affecting the depth of the convection zone and thus the efficiency of the dynamo mechanism. It would not affect M-type stars, who are thought to be fully convective.

\section{THE PHYSICS OF STELLAR FLARES}

A few flares were observed by XMM-Newton and were investigated spectroscopically and photometrically. In the previous section, I have summarized the abundance variations during flares, with respect to the non-flaring situation. Previous X-ray satellites already observed that a very hot $(\geq 10 \mathrm{MK})$ temperature component developed in the early phases of flares (e.g., Franciosini et al., 2001). Audard et al. (2001b) were able to follow the development of a hot thermal bremsstrahlung continuum in the high-resolution RGS spectra of a flare in HR 1099 and observe flux increase in the highly ionized Fe XXIII-XXIV lines formed at high temperatures. The emission measure distribution varied significantly at high temperatures, while at lower temperatures, it was consistent with the quiescent distribution. A large flare in YY Gem also displayed a high temperature (Güdel et al., 2001b). A subsequent observation of YY Gem also showed several flares in which the temperature slightly increased (Stelzer et al., 2002). The flare spectrum of AB Dor showed some temperature increase, which eventually fell back to the temperature of the hottest quiescent component (Güdel et al., 2001a).

Flare decay analyses were performed using an hydrodynamical approach (Peres et al., 1987; Reale et al., 1997). By studying the flare evolution in the $\log T-\log \mathrm{EM}$ plane, hints for significant heating during the decay phase were derived together with loop lengths of about $2 \times 10^{9} \mathrm{~cm}$ for YY Gem (Stelzer et al., 2002), and $5 \times 10^{10} \mathrm{~cm}$ for AB Dor (Güdel et al., 2001a). These lengths are about $60 \%$ of the stellar radius. Gondoin et al. (2002) finds an upper limit of $2 \times 10^{11} \mathrm{~cm}$ for a flare on FK Com.

The chromospheric evaporation scenario has been tested in flares in the M-type dwarf Proxima Centauri (Güdel et al., 2002b) and in the RS CVn binary $\sigma$ Gem (Güdel et al., 2002c). In this scenario, a beam of accelerated electrons travels along the magnetic fields from the flare reconnection site down to the lower atmospheric layers. While electrons with large pitch angles radiate gyrosynchrotron emission in the radio, electrons with small pitch angles impact in the dense chromosphere and emit hard X-rays $(>20 \mathrm{keV})$ through Coulomb collisions. The upper layer of the chromosphere is heated and lifted up to the corona by a pressure gradient, filling the coronal loops and cooling through soft X-ray radiation and conduction (e.g., Antonucci et al., 1984). Therefore, the radio (and hard $\mathrm{X}$-ray) light curve reflects the "power" injected into the chromosphere, while the soft X-ray light curve is a tracer of the accumulated energy after evaporation. A characteristic time dependence thus relates the two light curves: the derivative of the soft X-ray light curve resembles the shape of the radio (and hard X-ray) light curve ("Neupert effect", Neupert, 1968), as observed in solar impulsive flares. The optical $U$ band can also be used as a proxy of the radio light curve, since they are often quasi-simultaneous. Figure 4 shows the soft X-ray light curve and the optical $U$-band curve (panel a), and the soft X-ray derivative (panel b) for the flare in Prox Cen (Güdel et al., 2002b), consistent with a Neupert effect. A second X-ray peak occurred during the primary decay. Line ratios measured in the He-like $\mathrm{O}$ VII triplet showed significant electron density increases in the cool $(\approx 2 \mathrm{MK})$ plasma. The density jumped from $\approx 10^{9} \mathrm{~cm}^{-3}$ in quiescence to $\approx 4 \times 10^{11} \mathrm{~cm}^{-3}$ during the flux maxima, and fell back to $\approx 2 \times 10^{10} \mathrm{~cm}^{-3}$ during the decays. Güdel et al. (2002b) derived estimates of masses and volumes of the O VII emitting plasma for the primary peak, its decay, the secondary peak, and its decay, $M \approx 4.8 \times 10^{14} \mathrm{~g}, 2.1 \times 10^{15} \mathrm{~g}, 3.2 \times 10^{14} \mathrm{~g}$, and $2.5 \times 10^{15} \mathrm{~g}$, 


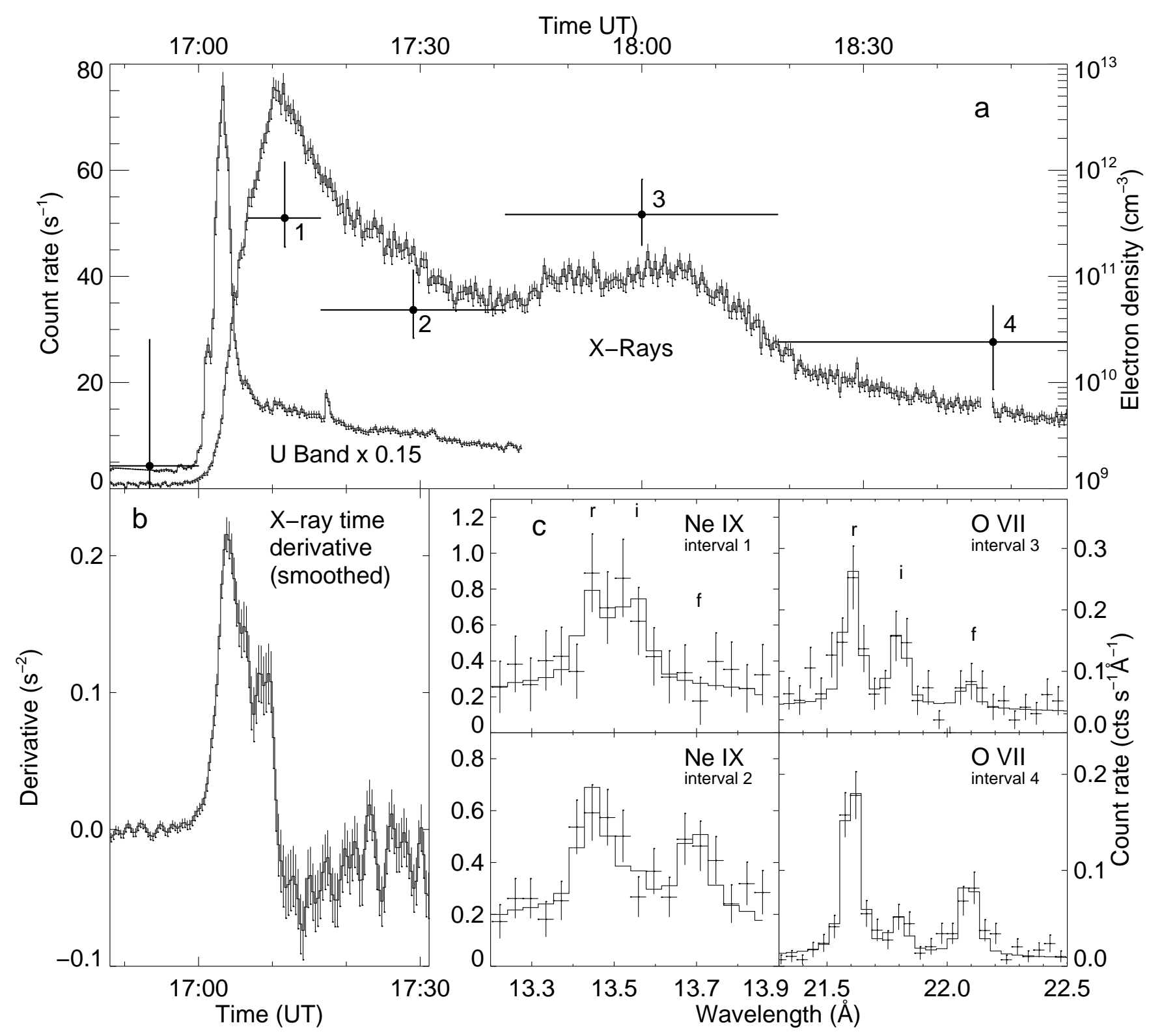

Fig. 4. A large flare in Proxima Centauri (Güdel et al., 2002b). Panel a: Soft X-ray and optical $U$-band light curves. Electron density measured in the $\mathrm{O}$ VII He-like triplet are shown as crosses. Panel $b$ : The time-derivative of the soft X-ray light curve resembles the optical curve, a signature of the Neupert effect. Panel c: He-like triplets during the four time intervals showed in panel a, showing the density increases during intervals 1 and 3. Used with permission of the American Astronomical Society.

respectively, while $V \approx 7.0 \times 10^{26} \mathrm{~cm}^{3}, 2.3 \times 10^{28} \mathrm{~cm}^{3}, 4.3 \times 10^{26} \mathrm{~cm}^{3}$, and $5.3 \times 10^{28} \mathrm{~cm}^{3}$, respectively. Thus interestingly, the plasma content (at temperatures close to the formation temperature of O VII) increased during the decays. The Neupert effect was also detected in a giant flare in $\sigma \mathrm{Gem}$, this time using the radio curve to trace the high-energy electrons (Güdel et al., 2002c). The total energy injected by these particles $\left(10^{33}-10^{36} \mathrm{ergs}\right)$ could satisfy or even exceed the total radiated energy $\left(4 \times 10^{34} \mathrm{ergs}\right)$, providing strong support for the chromospheric evaporation scenario. These observations show that coronal heating mechanisms, in this case the chromospheric evaporation scenario, can be applied in analogy to the Sun, even in stellar systems with coronae which may differ significantly from the Sun's. 

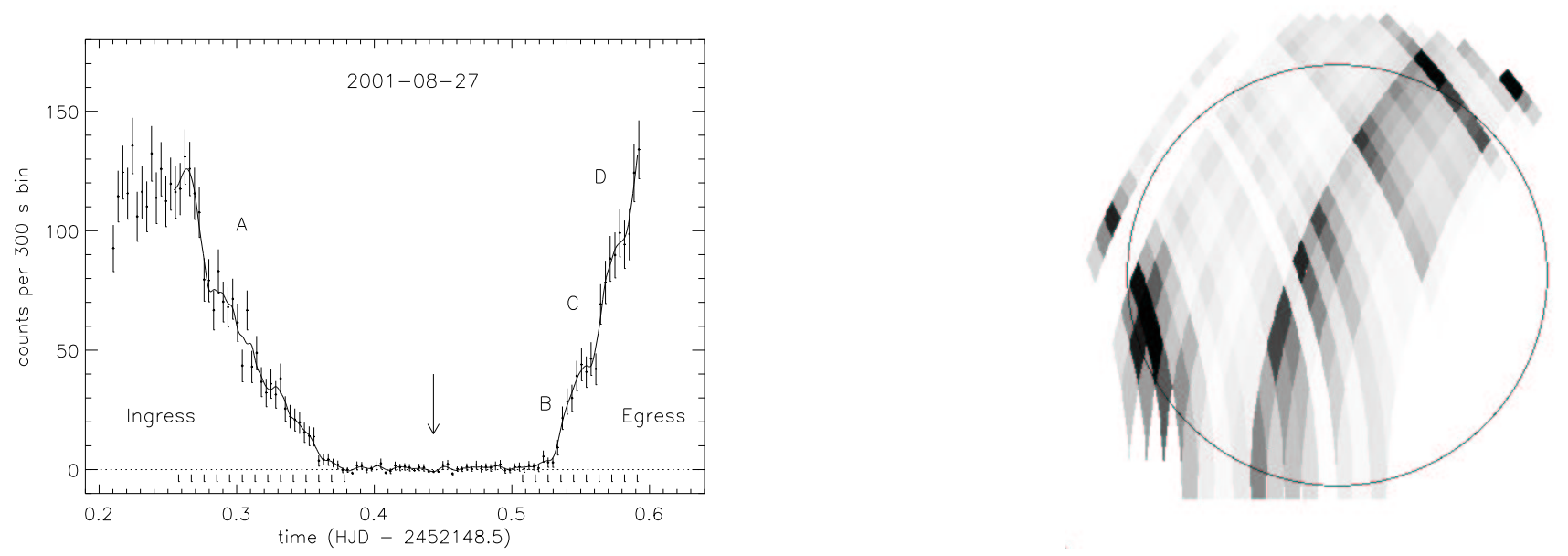

Fig. 5. Left: Light curve of the eclipse of the magnetically active G5 star behind the X-ray dark A0 star. Note the strong asymmetry of the light curve, and the individual steep flux decreases and increases, labeled $A$, B, C, and D. Right: A realization of a reconstructed map of the X-ray corona of the G-type star. From Güdel et al. (2003), used with permission of Astron. Astrophys.

\section{CORONAL STRUCTURES}

Coronal structures have been derived successfully from previous observations. It was established that stellar coronae have continuous emission measure distributions with significant plasma at high temperatures $(6-20 \mathrm{MK})$, in contrast with the cooler corona in the Sun. Investigations of solar analogs of different ages showed a significant correlation between the X-ray luminosity and the hot temperature component ( $L_{\mathrm{X}} \propto T^{4.5}$; Güdel et al., 1997). This was interpreted as evidence of a decrease in the efficiency of high-temperature coronal heating as a solar analog ages and its rotation slows down. Densities were obtained from line ratios in density-sensitive Fe L-shell lines; high densities $\left(10^{12}-10^{13} \mathrm{~cm}^{-3}\right)$ were found in high-temperature plasmas, whereas in the cooler plasma, lower densities $\left(10^{9} \mathrm{~cm}^{-3}\right)$ were found (e.g., see Bowyer et al., 2000, for a review). Coronal structures could also be inferred from simple loop models (e.g, Rosner et al., 1978; van den Oord et al., 1997). Indirect images of stellar X-ray coronae were derived from light curve inversions of eclipsing systems. This method proved to be very powerful (e.g., White et al., 1990; Schmitt and Kürster, 1993).

New XMM-Newton observations have contributed to the understanding of the thermal coronal structure as well. Line ratios of opacity-sensitive Fe lines showed that the X-ray spectra of cool stars are generally compatible with laboratory measurements of collisional plasmas (Audard et al., 2003). Line ratios measured in He-like triplets generally showed low densities in the "cool" O VII triplet (HR 1099: $<10^{10} \mathrm{~cm}^{-3}$; Capella: $<10^{10} \mathrm{~cm}^{-3}$; AB Dor: $3 \times 10^{10} \mathrm{~cm}^{-3}$; YY Gem and Castor: $2 \times 10^{10} \mathrm{~cm}^{-3}$; Procyon: $10^{10} \mathrm{~cm}^{-3}$; FK Com: $6 \times 10^{10} \mathrm{~cm}^{-3}$ ). Numerous blends in the $\mathrm{Ne}$ and $\mathrm{Mg}$ triplets, together with the poor spectral resolution of the RGS at wavelengths of the Si triplet prevented reliable density measurements. However, a tentative high density $\left(\approx 10^{12} \mathrm{~cm}^{-3}\right)$ in the "hot" Mg triplet was obtained for Capella (Audard et al., 2001a). Some contamination by weak Ne lines is however possible. Chandra HETGS is certainly a better instrument for such He-like triplets at short wavelengths. The above XMM-Newton result suggests that the hotter coronal plasma may display higher densities, reminiscent of the scenario of the presence of two classes of loops. These results need, however, to be verified with future observations. From the Chandra side, a systematic study of electron densities can be found, e.g., in Ness et al. (2002).

Eclipse mapping from the YY Gem binary (Güdel et al., 2001b), consisting of two identical magnetically active dMe stars, showed that the temperature $(\log T=6.5-7.1 \mathrm{~K})$ derived from the density scale height $\left(1-4 \times 10^{10} \mathrm{~cm}\right)$ is compatible with the range obtained from spectroscopic results. The statistical distributions of densities showed that the high-density plasma has a small filling factor. However, since the luminosity scales with $n_{\mathrm{e}}^{2}$, the observed emission comes mostly from plasma with $\log n_{\mathrm{e}} \approx 9.7-10.5 \mathrm{~cm}^{-3}$, which agrees well with the density measured in the He-like triplets. Güdel et al. (2001b) also found that active regions are distributed between $\pm 50^{\circ}$ in latitude.

The total eclipse of the secondary component of the $\alpha$ Coronae Borealis binary was monitored by XMM-Newton (Güdel et al., 2003). The binary consists of an X-ray dark A0 star and a magnetically active G5 dwarf. Since the 
primary does not emit in the X-rays, this provides an additional strong constraint to the possible location of the X-ray emission: most of it must lie close the surface of the secondary. Güdel et al. (2003) used three reconstruction methods to derive X-ray brightness maps. All reconstructions revealed similar distributions of X-ray bright regions and large areas with little flux. Figure 5 shows the eclipse light curve (left panel) and a realization of an X-ray brightness map (right panel). The electron density distributions ranged between $10^{9}-3 \times 10^{10} \mathrm{~cm}^{-3}$. During the total eclipse, the residual luminosity (either due to the A-type star or to an extended component of the coronae of the G dwarf) could not exceed $6 \times 10^{26} \mathrm{erg} \mathrm{s}^{-1}$.

\section{CONCLUSIONS}

Two years after its launch, XMM-Newton has produced exciting new results. The elemental composition of stellar coronae has played a central role with the discovery of an inverse FIP effect in most active stars. Detailed studies of solar analogs of different ages and activity levels revealed the presence of a transition from the inverse FIP effect to a solar-like FIP effect as age or magnetic activity level decreases. The inverse FIP effect in RS CVn binaries (or its absence in the less active Capella) seems to fit well into this transition. However, the absence of a FIP-related bias in the coronal abundances of Procyon may cast some doubt on the universality of this scheme. This inactive star has a temperature close to the Sun and thus its coronal abundances should display a solar-like FIP effect. Systematic studies of abundances needs to come in future analyses. Furthermore, such studies would benefit from more recent and accurate measurements of the photospheric abundances in active stars.

Although the spectroscopic abilities of XMM-Newton may play a major role in coronal studies of stars, long uninterrupted light curves have proved to be equally important. Simultaneous observations with the optical telescope on board XMM-Newton or with the radio VLA helped detect solar-like Neupert effects in the RS CVn binary $\sigma$ Gem, and in the nearby Proxima Centauri. The chromospheric evaporation scenario proved to fit the observations well with the observations. Thanks to the simultaneous high-resolution spectroscopy with the RGS, density increases during the flare in Prox Cen could unambiguously be measured, together with increases of the mass and volume of the "cool" plasma responsible for the $\mathrm{O}$ VII triplet during the decay phases.

Line ratios from the He-like triplets indicated low densities $\left(10^{9}-10^{10} \mathrm{~cm}^{-3}\right)$ in the cool coronal plasma (2 MK); density measurements from other He-like triplets, in particular those formed at higher temperatures and sensitive to higher densities, are still, however, uncertain, despite the tentative high-density value measured in the $\mathrm{Mg}$ triplet in Capella. However, some caution is in order; density measurements can be biased by unaccounted blends, or inappropriate treatment of the underlying continuum. Furthermore, He-like triplets formed at higher temperatures are sensitive to higher densities than triplets formed at lower temperatures. An inaccurate treatment of the triplet line fluxes could thus lead to high densities. Nevertheless, it is necessary to remind that electron densities only give a crude estimate of the actual densities in a stellar corona. Solar images and eclipse mapping methods have shown how inhomogeneous a corona is. The measurement of electron densities from line ratios thus must be understood in terms of an integrated average of a wide range of densities that are probably not distributed homogeneously.

The potential of XMM-Newton data has not yet been fully exploited. In the (recent) future, new results will undoubtedly be produced that may change our views of stellar coronae.

\section{ACKNOWLEDGMENTS}

I acknowledge support from the Swiss National Science Foundation (fellowship 81EZ-67388). I thank M. Güdel for providing figure material. M. Güdel, S. Kahn, and F. Paerels are also thanked for reading the manuscript and providing useful comments. I finally thank the referees, J. Linsky and one anonymous expert, for their useful suggestions that improved this review. This paper is based on observations obtained with XMM-Newton, an ESA science mission with instruments and contributions directly funded by ESA Member States and the USA (NASA).

\section{REFERENCES}

Antonucci, E., A. H. Gabriel, B. R. Dennis, The energetics of chromospheric evaporation in solar flares, Astrophys. J., 287, 917-925, 1984.

Arnaud, K. A., XSPEC: The first ten years, in Astron. Soc. Pac. Conf. Ser. 101, Astronomical Data Analysis Software and Systems V, edited by G. Jacoby and J. Barnes Astron. Soc. Pac., San Francisco, 17-20, 1996.

Audard, M., M. Güdel, and R. Mewe, The XMM-Newton view of stellar coronae: flare heating in the coronae of HR 
1099, Astron. Astrophys., 365, L318-L323, 2001 b.

Audard, M., E. Behar, M. Güdel, et al., The XMM-Newton view of stellar coronae: high-resolution X-ray spectroscopy of Capella, Astron. Astrophys., 365, L329-L335, 2001a.

Audard, M., M. Güdel, A. Sres, A. J. J. Raassen, and R. Mewe, A study of coronal abundances in RS CVn binaries, in press, Astron. Astrophys., 2003.

Bowyer, S., J. J. Drake, and S. Vennes, Extreme Ultraviolet astronomy, Annu. Rev. Astron. Astrophys., 38, 231-288, 2000.

Brinkman, A. C., E. Behar, M. Güdel, et al., First light measurements with the XMM-Newton reflection grating spectrometers: evidence for an inverse first ionisation potential effect and anomalous Ne abundance in the coronae of HR 1099, Astron. Astrophys., 365, L324-L328, 2001.

Catura, R. C., L. W. Acton, and H. M. Johnson, Evidence for X-ray emission from Capella, Astrophys. J., 196, L47-L49, 1975.

Drake, J. J., J. M. Laming, and K. G. Widing, Stellar coronal abundances. II: The first ionization potential effect and its absence in the corona of Procyon, Astrophys. J., 443, 393-415, 1995.

Drake, J. J., J. M. Laming, and K. G. Widing, Stellar coronal abundances. V. Evidence for the first ionization potential effect in alpha Centauri, Astrophys. J., 478, 403-416, 1997.

Drake, J. J., N. S. Brickhouse, V. Kashyap, et al., Enhanced noble gases in the coronae of active stars, Astrophys. J., 548, L81-L85, 2001.

Drake, J. J., Coronal spectroscopy and abundances with Chandra, Adv. Space Res., this issue.

Feldman, U., Elemental abundances in the upper solar atmosphere, Physica Scripta, 46, 202-220, 1992.

Franciosini, E., R. Pallavicini, and G. Tagliaferri, BeppoSAX observation of a large long-duration X-ray flare from UX Arietis, Astron. Astrophys., 375, 196-204, 2001.

Gondoin, P., C. Erd, and D. Lumb, Structure and evolution of FK Comae corona, Astron. Astrophys., 383, 919-932, 2002.

Güdel, M., E. F. Guinan, and S. L. Skinner, The X-ray Sun in time: a study of the long-term evolution of coronae of solar-type stars, Astrophys. J., 483, 947-960, 1997.

Güdel, M., M. Audard, K. Briggs, et al., The XMM-Newton view of stellar coronae: X-ray spectroscopy of the corona of AB Doradus, Astron. Astrophys., 365, L336-L343, 2001a.

Güdel, M., M. Audard, H. Magee, et al., The XMM-Newton view of stellar coronae: coronal structure in the Castor X-ray triplet, Astron. Astrophys., 365, L344-L352, $2001 \mathrm{~b}$.

Güdel, M., M. Audard, K. W. Smith, E. Behar, A. J. Beasley, and R. Mewe, Detection of the Neupert effect in the corona of an RS Canum Venaticorum binary system by XMM-Newton and the Very Large Array, Astrophys. J., 577, 371-376, 2002c.

Güdel, M., M. Audard, S. L. Skinner, and M. I. Horvath, X-ray evidence for flare density variations and continual chromospheric evaporation in Proxima Centauri, Astrophys. J., 580, L73-L76, 2002 b.

Güdel, M., M. Audard, A. Sres, R. Wehrli, and R. Mewe, Elemental abundances in stellar coronae: long-term evolution from the inverse to the normal first ionization potential effect, submitted to Astrophys. J., 2002a.

Güdel, M., K. Arzner, M. Audard, and R. Mewe, Tomography of a stellar X-ray corona: $\alpha$ Coronae Borealis, in press, Astron. Astrophys., 2003.

Haisch, B., and J. H. M. M. Schmitt, Advances in solar-stellar astrophysics, Publ. Astron. Soc. of the Pacific, 108, 113-129, 1996.

Hénoux, J.-C., Models for explaining the observed spatial variation of element abundances - A review, Adv. Space Res., 15, 23-32, 1995.

Hénoux, J.-C., FIP fractionation: theory, Space Sci. Rev., 85, 215-226, 1998.

den Herder, J. W., A. C. Brinkman, S. M. Kahn, et al., The Reflection Grating Spectrometer on board XMM-Newton, Astron. Astrophys., 365, L7-L17, 2001.

Jansen, F., D. Lumb, B. Altieri, et al., XMM-Newton observatory: I. The spacecraft and operations, Astron. Astrophys., 365, L1-L6, 2001.

Kaastra, J. S., R. Mewe, and H. Nieuwenhuijzen, SPEX: a new code for spectral analysis of X and UV spectra, in $U V$ and X-ray Spectroscopy of Astrophysical and Laboratory, edited by K. Yamashita and T. Watanabe, Univ. Acad. Press, Tokyo, 411, 1996.

Laming, J. M., and J. J. Drake, Stellar coronal abundances. VI. The first ionization potential effect and $\xi$ Bootis A: 
solar-like anomalies at intermediate-activity levels, Astrophys. J., 516, 324-334, 1999.

Laming, J. M., Should you trust atomic data?, in Astron. Soc. Pac. Conf. Ser. 277, Stellar Coronae in the Chandra and XMM-Newton Era, edited by F. Favata and J. J. Drake, in press, Astron. Soc. Pac., San Francisco, 2002.

Linsky, J. L., Nonradiative activity across the H-R diagram - Which types of stars are solar-like?, Solar Phys, 100, 333-362, 1985.

Linsky, J. L., X-ray coronae of stars: recent results from Chandra, Adv. Space Res., this issue.

Mason, K. O., A. Breeveld, R. Much, et al., The XMM-Newton optical/UV monitor telescope, Astron. Astrophys., 365, L36-L44, 2001.

Mewe, R., X-ray spectroscopy of stellar coronae, Astron. Astrophys. Rev., 3, 127-168, 1991.

Mewe, R., J. S. Kaastra, and D. A. Liedahl, Update of MEKA: MEKAL, Legacy, 6, 16, 1995.

Mewe, R., X-ray spectroscopy of stellar coronae: history - present - future, Solar Phys., 169, 335-348, 1996.

Meyer, J.-P., Solar-stellar outer atmospheres and energetic particles, and galactic cosmic rays, Astrophys. J. Suppl. Ser., 57, 173-204, 1985.

Ness, J.-U., J. H. M. M. Schmitt, V. Burwitz, et al., Coronal density diagnostics with helium-like triplets: CHANDRALETGS observations of Algol, Capella, Procyon, epsilon Eri, alpha Cen A\&B, UX Ari, AD Leo, YY Gem, and HR 1099, Astron. Astrophys., 394, 911-926, 2002.

Neupert, W. M., Comparison of solar X-ray line emission with microwave emission during flares, Astrophys. J., 153, L59-L64, 1968.

Pallavicini, R., X-ray emission from stellar coronae, Astron. Astrophys. Rev., 1, 177-207, 1989.

Peres, G., F. Reale, S. Serio, and R. Pallavicini, Hydrodynamic flare modeling - Comparison of numerical calculations with SMM observations of the 1980 November 12 17:00 UT flare, Astrophys. J., 312, 895-908, 1987.

Phillips, K. J. H., R. Mewe, L. K. Harra-Murnion, J. S. Kaastra, P. Beiersdorfer, P., G. V. Brown, and D. A. Liedahl, Benchmarking the MEKAL spectral code with solar X-ray spectra, Astron. Astrophys. Suppl., 138, 381-393, 1999.

Raassen, A. J. J., R. Mewe, M. Audard, et al., High-resolution X-ray spectroscopy of Procyon by Chandra and XMMNewton, Astron. Astrophys., 389, 228-238, 2002.

Reale, F., R. Betta, G. Peres, S., Serio, and J. McTiernan, Determination of the length of coronal loops from the decay of X-ray flares I. Solar flares observed with YOHKOH SXT, Astron. Astrophys., 325, 782-790, 1997.

Rosner, R., W. H. Tucker, and G. S. Vaiana, Dynamics of the quiescent solar corona, Astrophys. J., 220, 643-665, 1978.

Rosner, R., L. Golub, and G. S. Vaiana, On stellar X-ray emission, Ann. Rev. Astron. Astrophys., 23, 413-452, 1985.

Schmelz, J. T., Why stellar astronomers should be interested in the Sun, Adv. Space Res., this issue.

Schmitt, J. H. M. M., M. Kürster, A spatially resolved X-ray image of a star like the Sun, Science, 262, 215-218, 1993.

Schmitt, J. H. M. M., R. A. Stern, J. J. Drake, and M. Kürster, CF Tucanae: another case of coronal MAD syndrome?, Astrophys. J., 464, 898-909, 1996.

Sciortino, S., G. Micela, F. Damiani, et al., XMM-Newton survey of the low-metallicity open cluster NGC 2516, Astron. Astrophys., 365, L259-L266, 2001.

Smith, R. K., N. S. Brickhouse, D. A. Liedahl, and J. C. Raymond, Collisional plasma models with APEC/APED: emission-line diagnostics of hydrogen-like and helium-like ions, Astrophys. J., 556, L91-L95, 2001.

Stelzer, B., V. Burwitz, M. Audard, et al., Simultaneous X-ray spectroscopy of YY Gem with Chandra and XMMNewton, Astron. Astrophys., 392, 585-598, 2002.

Strüder, L., U. Briel, K. Dennerl, et al., The European Photon Imaging Camera on XMM-Newton: The pn-CCD camera, Astron. Astrophys., 365, L18-L26, 2001.

Turner, M. J. L., A. Abbey, M. Arnaud, et al., The European Photon Imaging Camera on XMM-Newton: The MOS cameras, Astron. Astrophys., 365, L27-L35, 2001.

van den Oord, G. H. J., C. J. Schrijver, M. Camphens, R. Mewe, and J. S. Kaastra, EUV spectroscopy of cool stars. III. Interpretation of EUVE spectra in terms of quasi-static loops, Astron. Astrophys., 326, 1090-1102, 1997.

White, N. E., R. A. Shafer, A. N. Parmar, K. Horne, and J. L. Culhane, X-ray eclipse mapping of AR Lacertae, Astrophys. J., 350, 776-795, 1990.

E-mail address of M. Audard audard@astro.columbia.edu

Manuscript received 1 December 2002; revised 4 January 2003; accepted 9 January 2003 